\title{
A New and Fast Optimization Algorithm: Fox Hunting Algorithm (FHA)
}

\author{
Murat Onay \\ Erciyes University, Faculty of Aeronautical and Space Sciences, Turkey
}

\begin{abstract}
A new population-based search algorithm called the Fox Hunting Algorithm (FHA) is presented here. FHA is a general-purpose algorithm that can be applied to solve almost any optimization problem. The algorithm mimics the fox hunting activity which is formed and improved since 16th century. The algorithm performs some neighborhood searches combined with random search. It can be used for both combinatorial optimization and functional optimization.
\end{abstract}

Keywords-component; fox hunting algorithm, functional optimization, swarm intelligence

\section{INTRODUCTION}

Classical methods often face great difficulties in solving many complex multi-variable Optimization problems that abound in the real world. In order to overcome the short comings of traditional mathematical techniques, population based soft computing algorithms have been introduced over the last decades.

Several evolutionary or meta-heuristic algorithms have since been developed which combine rules and randomness mimicking natural behaviors of the animals. These behaviors include biological evolutionary processes (e.g., the evolutionary algorithm proposed by Fogel et al. [1], De Jong [2], and Koza [3] and the genetic algorithm (GA) proposed by Holland [4] and Goldberg [5]) and animal behaviors (e.g., the Ant Colony Optimization proposed by Dorigo et al. algorithm [6] and the Particle Swarm Optimization algorithm proposed by Eberthart and Kennedy [7], the Bees Algorithm proposed by Pham [8] , Artificial Fish Swarm Algorithm proposed by Li [9]).

In the last decades, these meta-heuristic algorithms, especially GA-based methods have been studied by many researchers to solve various optimization problems. That is clear meta-heuristic algorithms have great results in many engineering area.

The FHA is a new meta-heuristic optimization algorithm which simulates the fox hunting activity of the human beings. Fox hunting involves the tracking, chase, and sometimes killing of a fox. Trained foxhounds, other scent hounds, fell terrier or smooth fox terrier are used to chase the fox. A master of foxhounds follows the hounds on foot or on horseback. A group of unarmed followers led follow the master of foxhounds [10]. Human beings use horses to go faster. They use foxhounds for tracking. Also they use terriers to deep search in holes and hives.
Optimization algorithms usually have two search mechanisms. The first one is a global search mechanism and the second one is a local search mechanism. By the experience researchers knows that the local search mechanism must be modified while searching approaches to the solution. Because of that two search mechanisms are not efficient for real world problems. So in this optimization algorithm there are three search mechanisms for searching in a more efficient way. The first one is a global search mechanism; second one is a local search mechanism; third one is the deeper local search mechanism. They are similar to the fox hunting activity. The first search with horses is like global search in the optimization process; it is fast and searches bigger areas with biggest steps in the whole search mechanism. The second one with trained foxhounds is like local search; it is slower than horses but searches the areas better. The third one with terriers is like deeper local search; it has the smallest steps but searches really deeply. So the solution can be found most closely to the global best position as fast as possible. Next section describes fox hunting and FHA briefly.

\section{FHA ALGORITHM}

In this section there will be two sub parts. First one will describe fox hunting activity and second one will describe FHA.

\section{A. Fox Hunting Activity}

Fox hunting is an activity involving the tracking, chase, and sometimes killing of a fox, traditionally a red fox, by trained foxhounds or other scent hounds, and a group of unarmed followers led by a master of foxhounds, who follow the hounds on foot or on horseback [10]. Figure I shows a traditional Fox Hunting Activity.

Fox hunting originated in the 16th century in the form which was practiced legally until 2005 in Great Britain, but it also takes place all over the world, including in Australia, Canada, France, Ireland, Italy, and the United States [12, 13] In Australia, the term also refers to the hunting of foxes with firearms, similar to spotlighting or deer hunting. In much of the world hunting in general is understood to relate to any game animals or weapons (e.g., deer hunting with bow and arrow); in Britain and Ireland, "hunting" without qualification implies fox hunting (or other forms of hunting with houndsbeagling, stag hunting, mink hunting, drag hunting, or hunting the clean boot) as described here. 


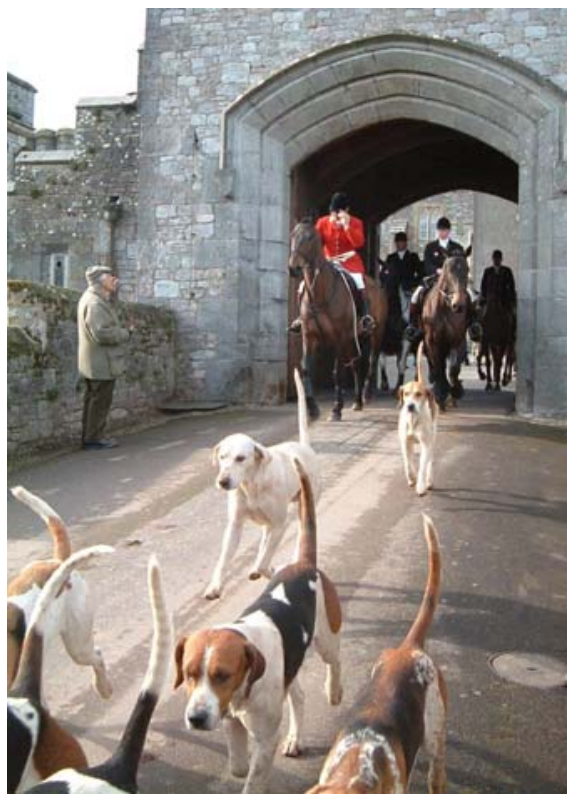

FIGURE I. MASTER OF FOXHOUNDS LEADS THE FIELD FROM POWDERHAM CASTLE IN DEVON, ENGLAND, WITH THE HOUNDS IN FRONT [11]

The sport is controversial, particularly in the UK, where its traditional form was banned in Scotland in 2002, and in England and Wales in November 2004 (law enforced from February 2005) [14], although certain modified forms of hunting foxes with hounds are still within the law and shooting foxes as vermin also remains lawful.

Proponents of fox hunting view it as an important part of rural culture and useful for reasons of conservation and pest control [15-17] while opponents argue that it is cruel and unnecessary [18].

\section{1) Animals of the Hunt}

Some animals are used for fox hunting for convenience. Major animals are usually horses, hounds and terriers. All three animals are described below.

\section{a) Horses}

The horses, called "field hunters" or hunters, ridden by members of the field, are a prominent feature of many hunts. Horses on hunts can range from specially bred and trained field hunters to casual hunt attendees riding a wide variety of horse. Hunters must be well-mannered, have the athletic ability to clear large obstacles such as wide ditches, tall fences, and rock walls, and have the stamina to keep up with the hounds.

Dependent on terrain, and to accommodate different levels of ability, hunts generally have alternative routes that do not involve jumping. The field may be divided into two groups, with one group, the First Field, that takes a more direct but demanding route that involves jumps over obstacles [19] while another group, the Second Field (also called Hilltoppers or Gaters), takes longer but less challenging routes that utilise gates or other types of access on the flat $[19,20]$.

\section{b) Hounds and other dogs:}

Fox hunting is usually undertaken with a pack of scent hounds [1], and in most cases, these are specially bred foxhounds [21]. These dogs are trained to pursue the fox based on its scent. The two main types of foxhound are the English Foxhound [22] and the American Foxhound [23]. It is possible to use a sight hound such as a greyhound or lurcher to pursue foxes [24], though this practice is not common in organized hunting, and these dogs are more often used for coursing animals such as hares [25].

Hunts may also use terriers to flush or kill foxes that are hiding underground [1], as they are small enough to pursue the fox through narrow earth passages.

\section{c) Birds of prey}

In the United Kingdom, since the introduction of the hunting ban, a number of hunts have employed falconers to bring birds of prey to the hunt, due to the exemption in the Hunting Act for falconry [26]. Many experts, such as the Hawk Board, deny that any bird of prey can reasonably be used in the British countryside to kill a fox which has been flushed by (and is being chased by) a pack of hounds [27].

\section{2) Hunting Procedure}

The hunt is often the setting for many social rituals, but the hunting itself begins when hounds are put into rough or brushy promising areas, where foxes often lay up during daylight hours. The pack moves to the promising areas by horses. If the pack manages to pick up the scent of a fox, they will track it for as long as they are able.

Scenting can be affected by temperature, humidity, and other factors. The hounds pursue the trail of the fox and the riders follow, by the most direct route possible.

Since this may involve very athletic skill on the part of horse and rider alike, fox hunting is the origin of traditional equestrian sports including steeplechase [28] and point to point racing [29].

The hunt continues until either the fox is overtaken, goes to ground (that is takes refuge in an underground burrow) or evades the hounds. In the UK, where the fox goes to ground, terriers may be entered into the earth to locate the fox so that it can be dug down to and killed, and this is carried out as a form of pest control [1].

\section{B. Proposed Fox Huting Algorithm (FHA)}

The FHA is a new and fast optimization algorithm in the field of swarm intelligence. The FHA is easy to write in any programing language. The FHA simulates superior behaviors of the animals while hunters searching fox. Swarm is made of initial population composed of individuals to search in the solution space. For example, we can simulate birds, ants, bees and other creatures in the particle swarm optimization, ant colony optimization, bees algorithm and artificial fish swarm algorithm. Here, in FHA, we use horses and different kinds of dogs as individuals for solving the problems.

In FHA, every individual has its own position composed of $\mathrm{D}$ (dimension of the problem) dimensions, a fitness value 
which represents the accommodation of the individual to the fitness function. The final solution would be the best position of the dogs especially terriers. The FHA keeps the best solution until it reaches the end of the iterations. Figure II shows the pseudo code for the algorithm in its simplest form.

In the fox hunting, real animals are used for tracking and chase. In the FHA, artificial animals are used for tracking and chase. These animals are horses, foxhounds and terriers respectively. Horses are used to move promising areas like rough or brushy areas in the hunting area. Then, foxhounds are used to pursue the fox. At the end, the fox goes to ground and terriers are used to enter into the earth to flush fox hidden underground and to locate the fox. In real world, horses move faster with bigger steps than dogs. Also, foxhounds moves faster than terriers with middle steps. Finally, terriers are used, so they move with smallest steps in the swarm. Similarly in the FHA, artificial horses (AH) move faster with bigger steps than artificial foxhounds (AF), and the AF move faster than terriers (AT). This behavior makes the FHA more effective.

1. Divide the $A H$ into two groups ( $A H 1$ and $A H 2)$ as in the real world

2. Randomly sprinkle $A H 1$ into M-dimensional solution space and randomly give values, which are in-range of boundaries.

3. Calculate the fitness of the $A H 1$

4. $\quad$ Choose better $m$ members of $A H 1$ and their positions $\left(m p_{i}\right)$ for neighborhood search

5. Calculate the promising weights of $m$ members $\left(p w m_{i}\right)$ by the equation (1)

6. The latest improvement obtained iteration number $(L I N)$ is set to 0 . Iteration number $I N$ is set to 0 .

7. While stopping criterion is not satisfied // Forming the new population

8. Randomly sprinkle $A H 2$ into M-dimensional solution space by the range of boundaries

9. Calculate the fitness of the $\mathrm{AH} 2$

10. Send the $A F$ to around the every $m p_{i}$ according to $p w m_{i}$

11. Calculate the fitness of the $A F$

12. Find $m$ best solutions between all $A F$ and $A H 2$

13. If any improvement is done in this iteration by $A H 2$ or $A F$ then $L I N=I N$

14. If $L I N<I N-7$ then make a sub-search:

a. $\quad$ send the $A T$ to around the $m p_{1}$;

b. calculate the fitness of the $A T$;

c. if best $A T$ is better than $m p_{1}$ then hold it as $m p_{1}$;

d. kill \%5 $A F$ (decrease the value of $A F$ to $0.95 A F)$;

e. re-calculate $p w m_{i}$

15. Increase $I N$; End While

FIGURE II. PSEUDO CODE OF THE BASIC FOX HUNTING ALGORITHM where $\mathrm{m}$ is number of promising solutions that are chosen from a group of solutions. mpi is position of the $\mathrm{m}$ members where $\mathrm{i}=1,2, \ldots, \mathrm{m}$. pwmi is an array that determines how many AF members will send to every mpi. pwmi can be writen as :

$$
p w m_{i}=\text { round }\left(\frac{A F}{m}+\frac{A F}{10 m}\left(\text { round }\left(\frac{m-2 i}{2}\right)\right)\right) \text { where } \mathrm{i}=1,2, \ldots,
$$

Here in Table I typical values of the parameters can be found. These parameters can be changed by experience on the problem and algorithm.

TABLE I. TYPICAL VALUES OF PARAMETERS FOR A SEARCH SPACE BETWEEN 0-20 (FOR EXAMPLE).

\begin{tabular}{|c|c|c|c|c|c|c|c|}
\hline Scalers: & $\boldsymbol{m}$ & $\boldsymbol{A H}$ & $\boldsymbol{A F}$ & $\boldsymbol{A T}$ & $\begin{array}{c}\boldsymbol{A H} \\
\text { Step } \\
\text { size }\end{array}$ & $\begin{array}{c}\boldsymbol{A F} \\
\text { Step } \\
\text { size }\end{array}$ & $\begin{array}{c}\boldsymbol{A T} \\
\text { Step } \\
\text { size }\end{array}$ \\
\hline $\begin{array}{c}\text { Typical } \\
\text { Values: }\end{array}$ & 10 & 300 & 100 & 100 & 20 & 2 & 0,2 \\
\hline
\end{tabular}

$\mathrm{AH}$ will be divided into two groups as been in the real world. First group, AH1, moves in the search area randomly at the beginning of the algorithm to find promising positions. Then AH1 followed by AF to find the way to the solution. AF starts with the promising positions of AH1. While AF makes neighborhood search in the promising areas, the second group, $\mathrm{AH} 2$, moves all around the search space to find a more promising area. The search of AH2 is like the global search in the optimization. Also search of AF is like the local search in the optimization. When AF can't find a better solution for about 7 iterations, AF gives his turn to AT. AT makes deeper search with smaller steps than AF.

\section{CONCLUSION}

In this paper, a new, fast, easy to write and efficient optimization algorithm is presented. The algorithm mimics the fox hunting activity which is formed and improved since 16th century. In the activity some animals are used by their superior features. This animals are horses (run faster with biggest steps in the activity), foxhounds (move after foxes by their smell facility with middle steps) and teriers (they are small so they can easily enter into the earth to locate the fox with smallest steps). Algorithm simulates these three animals with their superior features to find the optimal solution.

\section{REFERENCES}

[1] L.J. Fogel, A.J. Owens and M.J. Walsh, “Artificial Intelligence Through Simulated Evolution”, John Wiley, Chichester, UK, 1966.

[2] K. De Jong, "Analysis of the behavior of a class of genetic adaptive systems”, Ph.D. Thesis, University of Michigan, Ann Arbor, MI, 1975.

[3] J.R. Koza, "Genetic programming: a paradigm for genetically breeding populations of computer programs to solve problems", Rep. No. STANCS-90-1314, Stanford University, CA, 1990.

[4] J.H. Holland, “Adaptation in Natural and Artificial Systems”, University of Michigan Press, Ann Arbor, MI, 1975.

[5] D.E. Goldberg, "Genetic Algorithms in Search, Optimization and Machine Learning”, Addison Wesley, Boston, MA, 1989. 
[6] M. Dorigo, V. Maniezzo, A. Colorni, "Ant system: Optimization by a colony of cooperating agents", IEEE Transactions on Systems, Man, and Cybernetics - part B: Cybernetics 26, 29-41, 1996.

[7] R.C. Eberhart, and J. Kennedy, “A new optimizer using particle swarm theory", Proceedings of the Sixth International Symposium on Micro Machine and Human Science, Nagoya, Japan, 39-43. Piscataway, NJ: IEEE Service Center, 1995.

[8] D.T., Pham, A. Ghanbarzadeh, E. Koc, S. Otri, S. Rahim, and M. Zaidi, "The bees algorithm - a novel tool for complex optimisation problems," Proc 2nd Int Virtual Conf on Intelligent Production Machines and Systems (IPROMS 2006), Oxford, Elsevier, 2006.

[9] X.L. Li, “A New Intelligent Optimization-Artificial Fish Swarm Algorithm”, Doctor thesis, Zhejiang University of Zhejiang, China, 2003.

[10] Lord Burns, V. Edwards, J. Marsh, Lord M. Winter, "The Final Report of the Committee of Inquiry into Hunting with Dogs in England and Wales”, Her Majesty's Stationery Office, 2009.

[11] “Fox hunting”, https://en.wikipedia.org/wiki/Fox_hunting, Retrieved 27.01.2016.

[12] Griffin, Emma, “Blood Sport”. Yale University Press, 2007.

[13] “Fox hunting worldwide”, http://news.bbc.co.uk/2/hi/uk_news /428122.stm, BBC News. 1999-09-16, Retrieved 27.01.2016.

[14] "Hunt ban forced through Commons", http://news.bbc.co.uk/2/hi/uk_news/politics/4020453.stm, BBC News. 2004-11-19, Retrieved 27.01.2016.

[15] "Social impact of fox hunting on rural communities", http://www.mfha.org.uk/index.php?option=com_content\&task=view\&id $=62 \&$ Itemid=58, Masters of Fox Hounds Association. 2000, Retrieved 27.01.2016.

[16] "Creation and conservation of habitat by foxhunting", http://www.mfha.org.uk/index.php?option=com_content\&task=view\&id =64\&Itemid=56, Masters of Fox Hounds Association,27.01.2016.

[17] "The need for wildlife management", http://www.mfha.org.uk/index.php ?option=com_content\&task=view\&i $\mathrm{d}=65 \&$ Itemid $=54$, Masters of Fox Hounds Association, Retrieved 27.01.2016.

[18] “Campaign to Protect Hunted Animals", http://www.ifaw.org/international/node/6372, Retrieved 27.01.2016.

[19] "Information and Guidelines for Foxhunters in the Field", http://web.archive.org/web/20080308004907/http://www.independencef oxhounds.com/protocol.html, Independence Foxhounds. 2007, Retrieved 27.01.2016.

[20] "Hunting Hounds and Polo Ponies", https://web.archive.org/web /20071025165931/http://www.jocohistory.net/culture/11no3hunting.asp, JoCo History. 1998, Retrieved 27.01.2016.

[21] "English Foxhound History”, http://www.akc.org/dog-breeds/englishfoxhound/, American Kennel Club, Retrieved 27.01.2016.

[22] “English Foxhound Breed Standard”, http://www.akc.org/dogbreeds/english-foxhound/, American Kennel Club, Retrieved 27.01.2016.

[23] "American Foxhound Breed Standard", www.akc.org/breeds/american_foxhound/, American Kennel Club, Retrieved 27.01.2016.

[24] “Greyhound History”, http://www.akc.org/dog-breeds/greyhound/, American Kennel Club, Retrieved 27.01.2016.

[25] "End of the road for illegal hare coursing", http://www.bbc.co.uk/insideout/east/series7/hare_coursing.shtml, BBC Inside Out. 2005-01-24, Retrieved 27.01.2016.

[26] “The banned rode on”, http://www.theguardian.com/uk/2006/nov/ 07/hunting.ethicalliving, London: The Guardian, Retrieved 27.01.2016.

[27] I. McLeod, "Birds of prey and the Hunting Act 2004”, Justice of the Peace, 169: 774-775, 2005.

[28] “Steeplechase”, http://www.britannica.com/sports/steeplechase-horseracing, Encyclopaedia Britannica Online. 2008, Retrieved 27.01.2016.

[29] “A istory of Point to Point”, http://www.p2p.ie/content.php ?key=History, Irish Point to Point. 2003, Retrieved 27.01.2016. 